\title{
PFO closure consideration for refractory hypoxia and secondary prevention of recurrent arterial thromboembolism
}

\author{
David Maksimovich BS, Christopher M Merrick MD, Cihan Cevik MD
}

\begin{abstract}
Acute arterial and deep venous thrombosis presenting simultaneously is an uncommon medical emergency, usually secondary to an underlying cause. We report a 64-year-old woman with concurrent bilateral pulmonary emboli and acute thrombotic occlusion of the right brachial artery. Her work-up revealed a large patent foramen ovale (PFO) with a right to left intracardiac shunt and bilateral lower extremity deep venous thrombosis. The patient was unable to be weaned off mechanical ventilation due to her refractory hypoxia. However, after closure of the PFO the patient's oxygenation improved. This case demonstrates the potential beneficial role of PFO closure in a hypoxic patient with a right to left intracardiac shunt. In addition, closure of the PFO may provide secondary prevention of paradoxical systemic thromboembolism.
\end{abstract}

Keywords: patent foramen ovale, arterial and venous thrombosis, ischemic stroke, cryptogenic stroke, refractory hypoxia, right to left intracardiac shunt.

\section{INTRODUCTION}

Patent foramen ovale (PFO) is a congenital communication between the left and right atrium of the heart. Approximately 25 percent of the general population has a PFO. These are often incidentally identified on routine transthoracic $2 \mathrm{D}$ echocardiograms and can be confirmed by a transesophageal echocardiogram. Patent foramen ovales are associated with several clinical conditions, especially cryptogenic stroke in young patients. Closure of a PFO is currently not recommended in asymptomatic patients without previous cryptogenic stroke. ${ }^{1}$

There has been controversy whether PFO closure reduces rates of recurrent strokes. Based on the longterm randomized RESPECT trial findings (Recurrent Stroke Comparing PFO Closure to Established Current

Corresponding author: David Maksimovich Contact Information: Dmaksimovich@ucdavis.edu DOI: $10.12746 /$ swrccc.v7i31.579
Standard of Care Treatment trial), the Food and Drug Administration has now approved transcatheter closure of PFOs for the secondary prevention of recurrent stroke. ${ }^{2,3}$ According to this trial, the risk reduction for recurrent stroke is decreased by approximately 60 percent by PFO closure when compared to medical therapy alone. ${ }^{6}$ Other trials, such as CLOSE (Patent Foramen Ovale Closure or Anticoagulants versus Antiplatelet Therapy to Prevent Stroke Recurrence) and REDUCE (The Gore REDUCE Clinical Study), similarly demonstrated a risk reduction in recurrent strokes when compared to anti-platelet therapy alone. ${ }^{4,5}$ These studies were done with young and middle-aged patients under age 60. Furthermore, Tobis and coworkers demonstrated that PFO closure was effective in reducing oxygen requirements among 97 patients with systemic hypoxia and a right to left intracardiac shunt. ${ }^{7}$

\section{CASE}

A 64-year-old woman with no prior cardiac history was transferred from an outside hospital for severe 


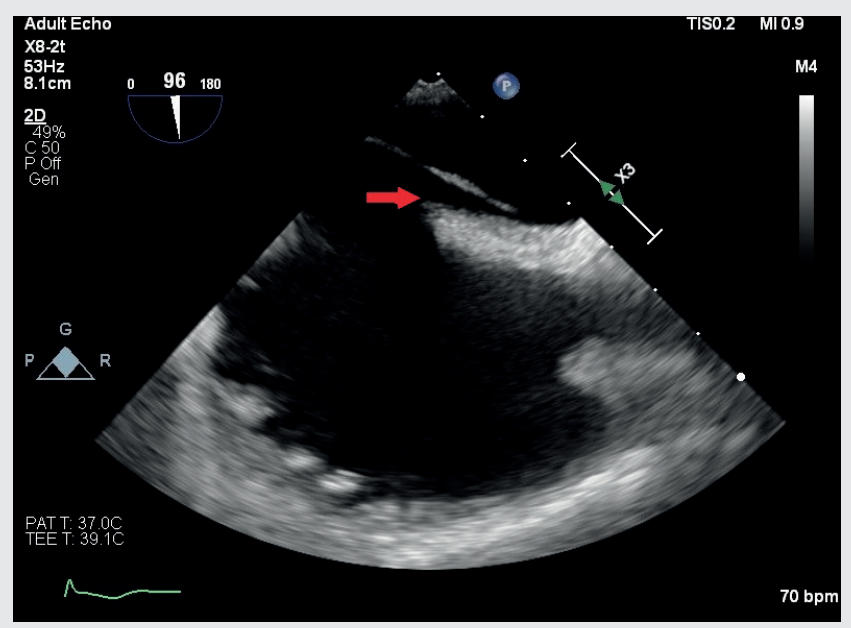

Figure 1. Transesophageal echocardiography (TOE) demonstrating the presence of a patent foramen ovale (PFO).

respiratory distress and the absence of a right upper extremity pulse. She was tachypneic on a Venturi mask with oxygen saturation in the 80 s. Initial vital signs in the emergency department included a blood pressure of $120 / 56 \mathrm{mmHg}$, a pulse of 89 beats per minute, a temperature of $36.6^{\circ} \mathrm{C}$, and a respiratory

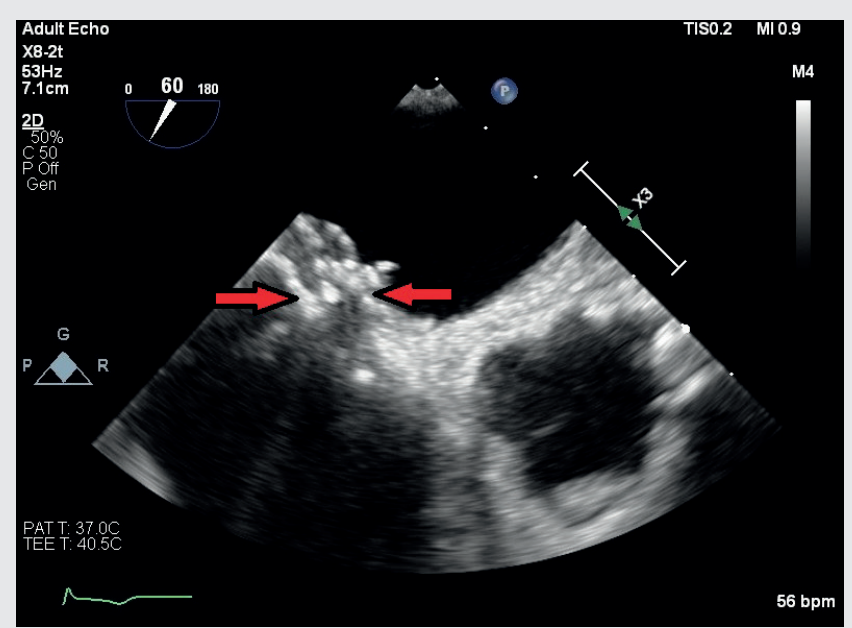

Figure 2. Prompt passage of contrast from right to left atrium through the PFO during injection of agitated saline contrast indicating a right to left intracardiac shunt. rate of 23 breaths per minute. On physical examination, the right upper extremity was cool and pulseless. Sensation in her right arm was mildly diminished, and she had flaccid weakness and pain of the right upper extremity. In addition, there was exquisite tenderness over both lower extremities with positive Homan's signs in both legs. She was alert and oriented to person, place, and time.

She was immediately taken into vascular surgery for arterial thrombectomy after confirmation of an arterial occlusion in the right brachial artery with ultrasound. Additional investigation revealed bilateral pulmonary emboli in the main segmental pulmonary arteries and deep venous thrombi in her lower extremities. On echocardiogram, a large size PFO was found creating a right to left intracardiac shunt (Figure 1 and 2). Further investigation for other causes of arterial thromboembolism and a hypercoagulable state did not demonstrate other hematological or medical disorders. This included work up for factor $\mathrm{V}$ Leiden mutation, antithrombin III deficiency, protein $\mathrm{C}$ deficiency, and acquired causes, such as malignancy and antiphospholipid antibodies.

The patient was started on a heparin drip. After the right upper extremity thrombectomy, the patient was admitted to the ICU while intubated. Multiple attempts to extubate the patient in the following three days failed, and she was unable to be weaned off ventilator support due to her refractory hypoxia and acute respiratory failure. The case was discussed extensively with the pulmonologist, the intensive care physician, the cardiologist, and her family. It was decided PFO closure might decrease the risk of future paradoxical embolisms and possibly improve her hypoxia likely due to her right to left intracardiac shunt. On day four, the patient underwent a percutaneous PFO closure using a $25 \mathrm{~mm}$ Gore PFO Occluder device (W. L. Gore \& Associates, Inc., Newark, DE) guided by transesophageal echocardiogram. The following day after PFO closure, the patient was able to be extubated. A repeat transthoracic echocardiogram showed Gore device in place and closure of her PFO (Figure 3). She remained stable with improved oxygenation saturation in the 90 s and was discharged home in three days. 


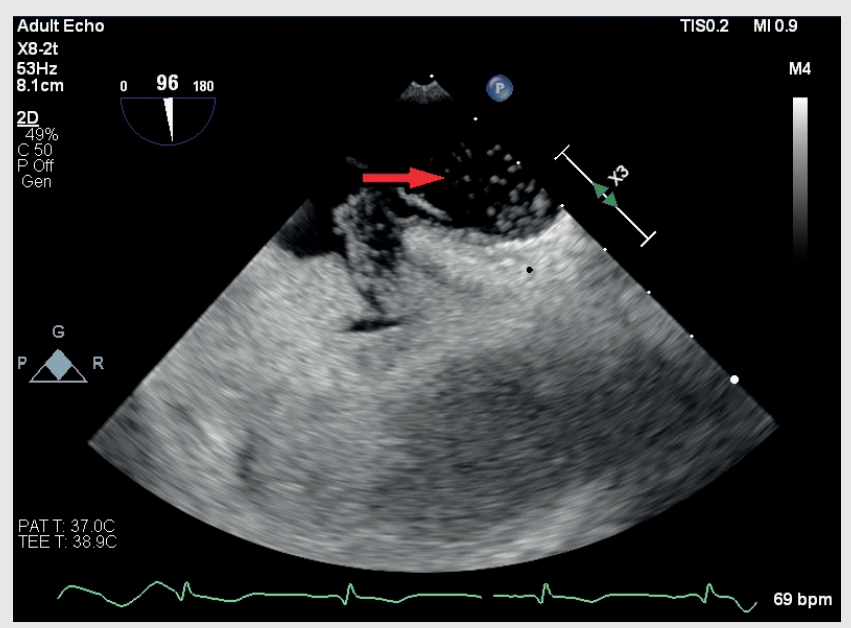

Figure 3. TOE showing PFO with a GORE occluder device in place.

\section{Discussion}

This report demonstrates the utility of PFO closure in patients with simultaneous systemic and venous thromboembolism. In addition, PFO closure may help improve hypoxia in intubated ICU patients with significant right to left intracardiac shunts.

Patent foramen ovale closure may possibly help patients with worsening hypoxemia due to lung diseases, such as severe obstructive sleep apnea, pulmonary fibrosis, and chronic obstructive pulmonary disease. Indications of a PFO in these patients would include dyspnea out of proportion to their underlying lung disease. However, PFO-mediated exacerbation of these conditions remains inadequately studied. ${ }^{8}$ The size of the right to left shunt and the severity of pulmonary disease likely determine the benefit of PFO closure. Our patient had no prior lung disease. However, she developed severe refractory hypoxia following a massive bilateral pulmonary embolism and could not be weaned from mechanical ventilation. Her arterial oxygen saturations improved dramatically following percutaneous PFO closure, and she was extubated the next day.

In summary, PFO investigation and possible closure should be considered in patients presenting with arterial and venous thromboembolisms to prevent future paradoxical embolic events. In addition, in patients with no known lung disease or known cause of hypoxia, a PFO should be ruled out as a potential cause of hypoxemia.

Article citation: Maksimovich D, Merrick CM, Cevik C. PFO closure consideration for refractory hypoxia and secondary prevention of recurrent arterial thromboembolism. The Southwest Respiratory and Critical Care Chronicles 2019;7(31):49-51

From: Rocky Vista University College of Osteopathic Medicine (DM), Parker, Colorado; Memorial Hospital Central (CMM, CC), Colorado Springs, Colorado

Submitted: 8/19/2019

Accepted: 10/8/2019

Reviewer: Scott Shurmur MD

Conflicts of interest: none

This work is licensed under a Creative Commons Attribution-ShareAlike 4.0 International License.

\section{REFERENCES}

1. Hagen PT, Scholz DG, Edwards WD. Incidence and size of patent foramen ovale during the first 10 decades of life: an autopsy study of 965 normal hearts. Mayo Clinic Proceedings 1984;59(1):17-20.

2. Parikh A, Vacek TP. PFO closure in high-risk patient with paradoxical arterial embolism, deep vein thrombosis, pulmonary embolism and factor $\mathrm{V}$ Leiden genetic mutation. Oxf Med Case Reports. 2018;2018(3): omx105. Published 2018 Mar 14. doi:10.1093/omcr/omx105

3. Saver JL, Carroll JD, Thaler DE, et al. Long-term outcomes of patent foramen ovale closure or medical therapy after stroke. N Engl J Med. 2017 Sep 14;377(11):1022-1032. doi: 10.1056/NEJMoa1610057. PubMed PMID: 28902590.

4. Mas JL, Derumeaux G, Guillon B, et al. Patent foramen ovale closure or anticoagulation vs. antiplatelets after stroke N Engl J Med 2017;377:1011-1021.

5. Furlan AJ, Reisman M, Massaro J, et al. Closure or medical therapy for cryptogenic stroke with patent foramen ovale. N Engl J Med 2012;366:991-9.

6. Carroll JD, Saver JL, Thaler DE, et al. Closure of patent foramen ovale versus medical therapy after cryptogenic stroke. N Engl J Med 2013;368:1092-100.

7. Tobis JM, Narasimha D, Abudayyeh I. Patent foramen ovale closure for hypoxemia. interventional cardiology clinics. 2017;6(4):547-554. doi: 10.1016/j.iccl.2017.05.003.

8. Mojadidi MK, Ruiz JC, Chertoff J, et al. Patent foramen ovale and hypoxemia. Cardiology in Review. 2019;27(1). doi:10.1097/CRD.0000000000000205. 